\title{
REVISTA DEL MUSEO DE LA PLATA
}

2016, Volumen 1, Número Especial: 111-118

"La Historia de la Geología en el Bicentenario de la Argentina"

\section{La Revista del Centro de Estudiantes del Doctorado en Ciencias Naturales}

Ruben J. Cucchi ${ }^{1}$

${ }^{1}$ Doctor en Ciencias Geológicas, Universidad de Buenos Aires. E-mail: rjcucchi@gmail.com

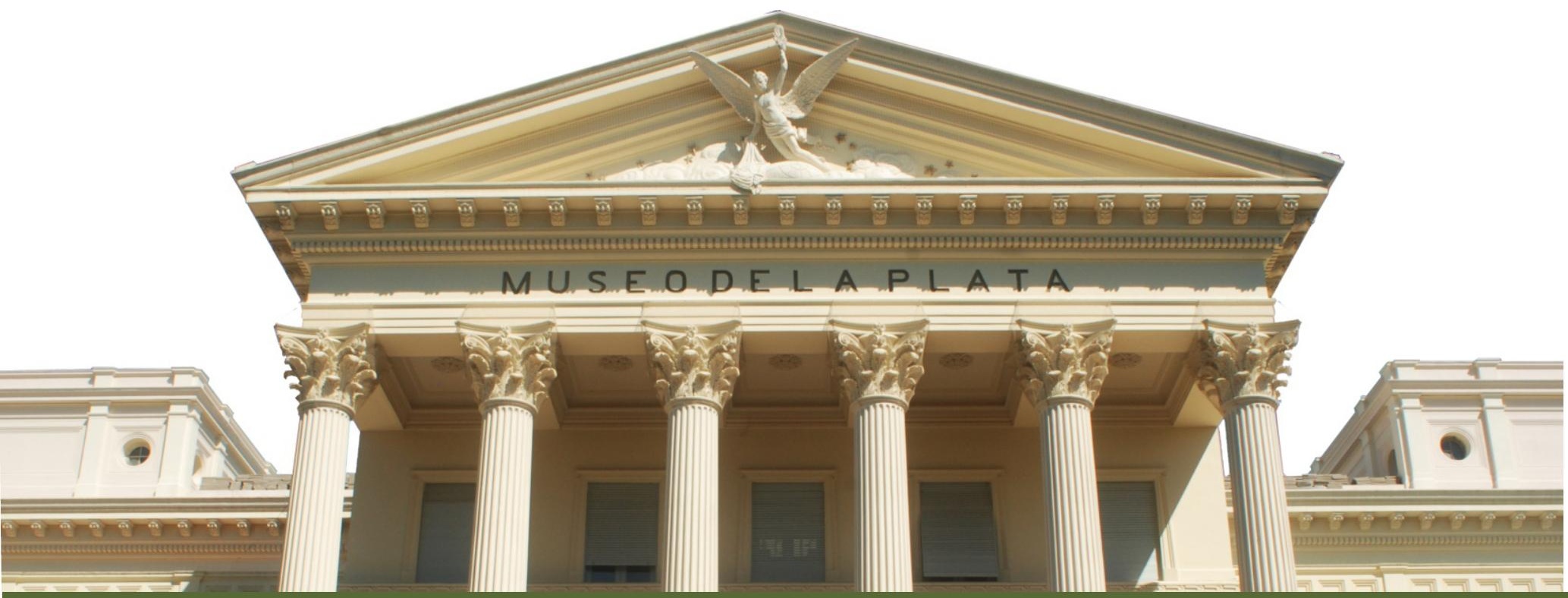




\title{
La Revista del Centro de Estudiantes del Doctorado en Ciencias Naturales
}

\author{
Ruben J. Cucchi ${ }^{1}$ \\ ${ }^{1}$ Doctor en Ciencias Geológicas, Universidad de Buenos Aires. E-mail: rjcucchi@gmail.com
}

\begin{abstract}
RESUMEN. La Revista del Centro de Estudiantes del Doctorado en Ciencias Naturales nació en mayo de 1935, una época en que en la Universidad se priorizaba el profesionalismo por sobre la academia. ¿Por qué considero de interés analizar la aparición de esta revista? En primer lugar no era usual en nuestro país la publicación de una revista científica en el ámbito de las Ciencias Naturales. Sirva de ejemplo que la revista de la Asociación Geológica Argentina recién aparece en 1945. En segundo lugar permitía dar a conocer a investigadores argentinos no sólo en nuestro país sino en el exterior y el intercambio de material bibliográfico con otras instituciones contribuyó a formar el patrimonio de la biblioteca del Centro de Estudiantes. A partir del año 1941 apareció con el nombre de "Holmbergia", en homenaje a Eduardo L. Holmberg "el primer profesor argentino nativo" y "prócer de nuestra patria" como dijera Jorge A. Crespo en el editorial del $n^{\circ} 6$ del tomo III. El análisis de las colaboraciones nos permite conocer a quienes fueron los primeros investigadores argentinos y contribuye al estudio de la historia de la ciencia en nuestro país. Esta revista, publicada con el aporte económico de alumnos, profesores y graduados tuvo dos etapas, desde su fundación en 1935 hasta 1946 y una segunda entre 1956 y 1961 cuando con el nº 17, cesó de publicarse.
\end{abstract}

Palabras clave: Universidad de Buenos Aires, Ciencias Naturales, Centro de Estudiantes, Revista, Holmbergia

\begin{abstract}
The Journal of the student center of the doctorate in natural sciences. The first issue of the journal of the Centro de Estudiantes del Doctorado en Ciencias Naturales appeared in May 1935, at a time when research was not too much encouraged at the University. The reasons of my interest in studying this event are various. In the first place, scientific publications were quite unusual at the time, e.g. the first journal of the Asociación Geológica Argentina was published in 1945. On the other hand it allowed local researchers to become known not only in our country but also abroad due to the exchange of bibliographical information with other institutions that gave rise to the Centro de Estudiantes library. In 1941 its name was changed to "Holmbergia" as a way to honor Eduardo Holmberg, "the first native Argentinian Professor" as stated by Jorge A. Crespo in the Editorial of number 6, Vol. III. Looking through the different issues it is possible to get acquainted with some of our first scholars and it turns out as a valuable contribution to obtain an idea of the history of science in our country. The magazine was sustained by the financial contribution of students, graduates and professors and it was published in two different periods, the first one from its beginning to 1946, and a second period from 1956 to 1971, when the last number was published.
\end{abstract}

Keywords: Buenos Aires University, Center of Natural Sciences Students, Jurnal, Holmbergia

*Trabajo presentado en el marco del IV Congreso Argentino de Historia de la Geología (IV CAHGEO) (La Plata, 15 y 16 de septiembre de 2016). 


\section{Introducción}

Es de interés analizar la aparición de una revista científica promovida por el Centro de Estudiantes del doctorado en Ciencias Naturales, (CEDCN, de cuya fundación el 29 de agosto de 1929 se cumplirán 87 años en 2016), creada cuando eran muy pocas las revistas dedicadas a dar a conocer el estado de las investigaciones que se realizaban en la Universidad de Buenos Aires, sobre todo en una época en que se priorizaba el profesionalismo por sobre la academia.

¿Cuál es el significado de la aparición de una revista cuya meta fue dar a conocer el estado de las investigaciones en el campo de las Ciencias Naturales y que además fue editada por un centro de estudiantes universitarios? ¿Constituía un hecho aislado?, ¿Cómo se relacionaba con la situación del país en ese momento? ¿Cómo era el clima universitario en los años 30 del siglo XX? ¿Y cuál el clima político? La universidad vivía bajo los postulados de la Reforma Universitaria de 1918 que había modificado profundamente la vida universitaria, en especial por la instalación del gobierno tripartito y el establecimiento de una enseñanza con mayor énfasis en la ciencia y el abandono de un saber escolástico como era el caso en Córdoba.

La Revista del Centro de Estudiantes del Doctorado en Ciencias Naturales nació en mayo de 1935 y en las palabras de su director Román A. Perez Moreau "en esta publicación debemos reflejar la investigación que se realiza en los laboratorios de nuestra escuela, y cuál es la participación que cabe al personal docente y a los alumnos. Pues no debemos olvidar que el Doctorado en Ciencias Naturales es una de las pocas carreras cientificas, por no decir la única de la Universidad de Buenos Aires". Esta afirmación que parece exagerada se puede explicar habida cuenta que Ameghino, Holmberg, Burmeister, Gallardo, entre otros científicos de la época, tenían gran predicamento tanto local como internacional y no pasaba lo mismo con otras disciplinas más volcadas al profesionalismo. Las ciencias naturales en la Argentina habían comenzado su desarrollo con la llegada de los científicos europeos, principalmente alemanes, traídos por Sarmiento a mediados del siglo XIX y cuyo objetivo fundamental fue completar e iniciar el conocimiento del territorio argentino. Esta tarea estuvo radicada inicialmente en Córdoba donde se fundó la Academia Nacional de Ciencias.

En cada una de los números de la Revista, aparte de las comunicaciones científicas se daban noticias sobre bibliografia, publicaciones recibidas por la Biblioteca del Centro, nuevos doctores en Ciencias Naturales, comentarios bibliográficos, lista de personas que colaboran con la financiación de la Revista, etc. etc. La importancia de la Revista se pone de manifiesto con los 114 países de Europa y América que recibieron Holmbergia en intercambio dando cuenta así del grado de desarrollo de un centro de estudiantes de una universidad argentina (Revista $n^{\circ} 12-13,1956$ ). La revista puede actualmente consultarse en la Biblioteca Digital de la Facultad de Ciencias Exactas y Naturales de la Universidad de Buenos Aires, www.digital.bl.fcen.uba.ar.

En lo que sigue se darán a conocer temas vinculados principalmente con la Geología que a juicio del autor son de la mayor importancia en lo que hace a la historia de las ideas geológicas imperantes en la Argentina en aquellos momentos o bien con temas de inocultable trascendencia.

\section{La Revista del CEDCN}

\section{Número 1 (1935)}

El primer número se abre con la conferencia que el Dr. Alberto Castellanos diera a pedido de la Comisión de Cultura del Centro de Estudiantes del Doctorado en Ciencias Naturales sobre la Vida y Obra de Linneo, a quien el autor considera el gran "reformador de las Ciencias Naturales" y atribuye el mérito de haber colocado al hombre entre los Primates y a diferencia de Bufón, quien concebía al 
ser humano como un cuarto reino, Linneo lo ubica como una especie del reino animal, creando el género y la especie Homo sapiens L. Según Castellanos "su perspicacia llega a lo inimaginable para la época (1766) ubicarlo entre los Primates como último término de la serie, es decir, los monos, tesis que con mucha posterioridad trataría magistral y novedosamente Darwin".

El aspecto relacionado con la docencia y con la extensión es abordado por I. Rafael Cordini en una colaboración sobre foraminíferos que es una guía práctica dedicada a la manipulación de los foraminíferos bentónicos con vista a principiantes en el estudio de estos microfósiles, de suma importancia en los estudios sedimentológicos relacionados con la búsqueda petrolera ya que permiten determinar cambios de facies, edad de los estratos e interpretar condiciones del depósito de los sedimentos.

La revista en su contratapa muestra la integración de la Comisión Directiva, de sus diversas comisiones internas: de Cultura (cuya secretaria era Delia Ingenieros), de Fiestas, de Biblioteca y una curiosa Comisión pro-modificación del plan de estudio, además de una Delegada a la Federación local (F.U.B.A.).

\section{Número 2 (1936)}

En el número 2, de junio de 1936 sobresale el trabajo de Pablo Groeber, un ensayo sobre la Paleoclimatología de la Argentina, quizás el primero en su tipo publicado en nuestro país, y se refiere a las "Oscilaciones del clima en la Argentina desde el Plioceno", al tiempo que considera que el edificio estructural de la Argentina se originó por las fuerzas orogénicas que tuvieron lugar en el Plioceno. Un predominio general de la destrucción mecánica sobre la química indicaría un descenso masivo de las precipitaciones y de la temperatura. La formación de una costra calcárea requiere un verano seco y caluroso y un invierno frío con precipitaciones de entre 300 y $600 \mathrm{~mm}$ anuales dando lugar a una mayor disolución de carbonato de calcio si hay un valor adecuado de $\mathrm{CO}_{2}$. El agua se carga con mayor concentración de $\mathrm{CO}_{2}$ cuanto más baja es la temperatura y en los veranos secos y calurosos hay evaporación en los niveles superiores; el agua que asciende por capilaridad y por efecto de la presión atmosférica al evaporarse se deposita el carbonato de calcio formándose así las costras calcáreas. En el oeste central y en la Patagonia septentrional la costra calcárea se extiende a grandes áreas llegando desde Mendoza a través de la Pampa central al suroeste de la provincia de Buenos Aires y en parte a la provincia de Corrientes. Esta situación es interpretada por Groeber como resultado de un cambio climático, que en la Patagonia se expresa en una extensa glaciación.

\section{Números 3 a 5 (1937 - 1941)}

En mayo de 1937 aparece el $n^{\circ} 3$ y Juan Keidel inicia un estudio de la Prepuna de Jujuy y Salta refiriéndose a las características orográficas, hidrográficas y climatológicas de esa región; se trata de una contribución inconclusa pues no continuará en ninguna otra entrega de la Revista.

El tomo II está constituido por los número 4 y 5 de abril de 1938 y 1939, respectivamente.

En el 4 se destaca la nota necrológica sobre Eduardo Ladislao Holmberg, en cuyo honor, como veremos en el número 6 del tomo III, se llamará la Revista. Se presenta la lista de publicaciones recibidas en canje y se inicia una sección dedicada a los nuevos Doctores en Ciencias Naturales: una paleontóloga la Dra, Noemí Cattoi y una geóloga la Dra. María Elisa Hermitte de Nogués.

Holmberg (1852-1937), graduado en Medicina en 1880 con una tesis "El fosfeno", había desarrollado un especial interés por las Ciencias Naturales, lo cual fue puesto de manifiesto en varias publicaciones, entre ellas "Una excursión por el río Luján" aparecida en "El Naturalista Argentino". Fue fundador y director del Jardín Zoológico municipal e incursionó en diversos campos de la Zoología, como mamíferos, aves, peces, moluscos, arácnidos e insectos, y de la Botánica: Flora de la 
República Argentina, aparecida en el censo de 1895. Fue el primer profesor argentino que ocupó cátedras de Ciencias Naturales en la Facultad de Ciencias Exactas, Físicas y Naturales de la Universidad de Buenos Aires. Su prédica inclaudicable fue por la causa de todos los tiempos: la libertad de las ideas.

En el número 5 Horacio J. Harrington presenta un trabajo sobre los Trilobites, una clase de artrópodos enteramente desaparecida; se refiere a su organización y estructura y sus relaciones con los demás Arthropoda. Enrique Fossa-Mancini mucho antes de la aparición de los GPS presenta un erudito estudio sobre "la Orientación por medio del Sol" destinado a naturalistas que desean determinar la posición del observador en relación con los objetos que se ven alrededor.

Esther Wahnisch da noticias del perfil geológico y contenido de fósiles en la Perforación Riachuelo $\mathrm{n}^{\circ} 5$, ubicada en González Catán, efectuada con motivo de trabajos de rectificación del cauce del río Matanzas. La lista de doctorados en Ciencias Naturales se amplía a 9, entre biólogos y geólogos.

En un apéndice del Número 5, aparecido en julio de 1941, se incluyen las "Observaciones geológicas en el cerro Valdivia”, como homenaje a su autor, el Dr. Blas V. Alascio, fallecido en el ejercicio de su profesión.

\section{Número 6 (1941)}

Con el Número 6 del tomo III comienza una etapa de la revista bajo un nuevo nombre: HOLMBERGIA. Lleva este nombre en homenaje al "primer profesor argentino nativo catedrático de las ciencias naturales, profesor preclaro de gran influencia cultural en su época, de espíritu siempre juvenil y prócer civil de nuestra patria", tal cual expresa la nota del director de la Revista Jorge A. Crespo dirigida a los lectores con la que se abre el tomo.

En este número se incluye el artículo de Groeber sobre el desarrollo del drenaje en América del Sur, cuyo diseño está relacionado con acciones tectónicas, especialmente de ascenso y descenso, relleno de cuencas y erosión concurrente; presenta así un mapa de regiones y líneas estructurales para vincular todos estos procesos

Hay una nota necrológica sobre Fernando Lahille firmada por el Dr. Alberto E. J. Fesquet con el subtítulo de "La aplicación de las Ciencias Naturales a la colonización y a la defensa de los intereses agropecuarios del país", cuya sola enunciación es una declaración de principios y ubica a finales del siglo XIX y comienzos del siguiente la, al parecer eterna, dicotomía entre ciencia pura y ciencia aplicada. "Estimar las ciencias naturales en proporción a la aplicación incesante a la satisfacción de las necesidades materiales, sería rebajarlas; es necesario también y ante todo buscar en ellas, la satisfacción de nuestros deseos espirituales y de nuestra sed de saber".

\section{Número 7 (1944)}

En el $n^{\circ} 7$ del tomo III siguen las contribuciones de Román Pérez Moreau, Martín Doello-Jurado, y Armando F. Leanza. La del primero de estos autores trata sobre la fitogeografía de la Provincia Antartándica. Se trata de un resumen de un trabajo presentado al X Congreso Científico Chileno, en el que se exponen las discrepancias del autor acerca de los conceptos de "Selva" y "Formación" fundamentando sus ideas al respecto. A su vez Martín Doello-Jurado en "Conceptos y palabras en Ciencias Naturales" se refiere a por qué es preferible en Geología el uso de "Cuartario" y no "Cuaternario" ya que este último término se refiere a una cosa compuesta de otras cuatro, así como en Química lo hace a compuestos formados por cuatro elementos. El número es cerrado con la contribución de Armando F. Leanza: "Introducción al estudio de la Paleontología" centrado en el 
estudio de los pelecípodos, una clase homogénea dentro de los Moluscos que continua en el número 8 del tomo IV.

\section{Números 8 y 9 (1945 y 1945)}

En el tomo IV, cambia la portada de la revista; se cumplen 10 años de su aparición y en el $n^{\circ} 8$ los trabajos presentados pertenecen a Alberto Castellanos, Alberto E.J. Fesquet, Román Pérez Moreau, Armando Leanza, Manuel Barros, Pascual Sgrosso, Arnold Heim y Harold Moldenke. El número se abre con un trabajo de Alberto Castellanos sobre "Las exploraciones botánicas en la época de la Independencia, 1810-1853" pero se centra en la figura de John Tweedie un escocés que fue contratado en 1825 por los hermanos Parish Robertson para que viniera a dirigir las obras de jardinería de la estancia Santa Catalina que integraba la colonia escocesa de Monte Grande en la provincia de Buenos Aires; no llegó solo sino acompañado por 100 colonos.

La "Nota fisiográfica sobre las cuencas cerradas", de Pascual Sgrosso, se refiere a los bolsones, explayándose sobre las condiciones para su génesis; agrega una clasificación del territorio argentino según cuencas indicando que un $46,90 \%$ del territorio no posee ningún desagüe con un total de más de 1.3 millones de $\mathrm{Km}^{2}$.

Arnold Heim, un afamado geólogo suizo, dio una conferencia en octubre de 1944 en la Facultad de Ciencias Exactas, Físicas y Naturales de la U.B.A., a pedido del Centro de Estudiantes del Doctorado en Ciencias Naturales, la que aparece publicada en Holmbergia bajo el nombre de "El perfil tectónico del Himalaya Central". Las secciones de Bibliografía, movimientos de la biblioteca del Centro y la nómina de las personas que contribuyen al sostén de la Revista ponen fin al contenido de este número que celebra el décimo aniversario de Holmbergia.

En el $n^{\circ} 9$ del tomo IV se presentan artículos de estudiantes, uno que recién comienza su carrera y otro que la finaliza. El último es de Silvia E. Morales Gorleri de Tribiño y es un resumen de su tesis "La causalidad y el determinismo en la biología" publicado en Imprenta Coní en 1946, y se presenta en la Revista bajo el nombre de "La biología evolucionista del siglo XIX".

Lucas Kraglievich presenta "Notas paleontológicas. Resultados de una excursión a Monte Hermoso y zonas vecinas" con varios perfiles de las barrancas de Monte Hermoso, provincia de Buenos Aires.

\section{Números 10 y 11 (1956)}

El número 10 del tomo $\mathrm{V}$, de febrero de 1956 marca la reaparición de Holmbergia luego de 10 años. También marca un cambio en la presentación de la revista con un diseño más actualizado y un notable aumento en los avisos publicitarios, buscando financiar su publicación. La esperanza es que su aparición sea trimestral, deseo expresado por su director en su nota "A los lectores".

El número da comienzo con una novedad en el país como es la presentación de "Nociones de micropaleontología" de Horacio H. Camacho, una introducción a dicha disciplina que comienza a desarrollarse en la Argentina. Se incluyen las "Tesis de doctorado (Período 1946-1955)" con 50 registros de tesis.

En marzo de 1956 aparece el $n^{\circ} 11$ con un trabajo de Jorge F. Villar Fabre, que constituye el primer trabajo de mineralogía aparecido en Holmbergia, en el que se describe una arcilla, Alillidromica, recurriendo para tal fin a análisis químicos y utilizando rayos X. Otro trabajo mineralógico es el de Roberto J. Poljak que aporta "una nota sobre antigorita y crisotilo de la Quebrada Yesera, Mendoza" usando para su determinación técnicas de microscopía electrónica y diagramas de rayos X. 
Armando F. Leanza se ocupa del "mutualismo entre un Ermitaño y un Briozoario fósiles, cohabitantes en la conchilla de un caracol”. Según el autor se trataría de un caso de mutualismo más bien que de simbiosis como fuera postulado por otros autores.

\section{Números 12-13 (1956)}

El tomo V finaliza con un número doble el 12-13 que aparece en agosto-noviembre de 1956, con colaboraciones de Raúl A. Ringuelet, Osvaldo A. Reig, Ricardo O. Gutiérrez y Marcelo R. Irigoyen. El artículo de Ringuelet versa sobre "Ambientes acuáticos continentales. Ensayo Bioecológico con particular aplicación a la República Argentina" con 121 referencias bibliográficas; se trata de un verdadero curso y puesta al día sobre dichas disciplinas.

El trabajo de Osvaldo Reig es "Sobre la posición sistemática de Zygolestes paranensis Amegh. y de Zygolestes entrerrianus Amegh., con una reconsideración de la edad y correlación del "Mesopotamiense"

Se presenta por vez primera una sección de Extensión Didáctica con una contribución de Marcelo R. Irigoyen: "La aerofotografía y la fotointerpretación. Necesidad de su enseñanza en nuestras Universidades" explicando la utilización de esta tecnología en aplicaciones generales, topográficas, geológicas, de planificación, urbanismo, agronomía, geología, etc.

La lista de instituciones que reciben Holmbergia alcanza a 114, de diferentes países americanos y europeos, lo que da cuenta de la importancia que ha llegado a tener esta revista de un centro de estudiantes de una universidad argentina.

\section{Número 14 (1959)}

El número 14 del tomo es de marzo de 1959. Esta entrega tiene un especial significado pues al cumplirse los 100 años de la aparición del Origen de las especies de Carlos Darwin el número está dedicado a problemas de evolución biológica. Se abre con un artículo de E.H. Rapoport sobre "Problemas acerca del origen de la vida" que, como su nombre indica, cubre un aspecto muy general de un sistema biogenerativo, mientras que el de Jorge Wright se circunscribe a "la evolución del basidio". La entrega de Osvaldo Reig es acerca de "La celebración Darwinista. Antecedentes nacionales y responsabilidad presente" con un análisis crítico de la situación actual de la ciencia en la Argentina, que incluye la participación de Ameghino, Holmberg y aún Sarmiento en la difusión y esclarecimiento sobre el evolucionismo.

El botánico Víctor García publica sobre "Embriología y Filogenia" y de cómo el uso de datos embriológicos se utiliza para solucionar algunos problemas que se presentan en taxonomía filogenética.

Las "Nuevas ideas sobre megaevolución" es desarrollada por Kostantin Gavrilov refiriéndose a un artículo de Marcus de 1958 sobre "The evolution of the Animal Phyla", en el que compara el nuevo sistema propuesto por Marcus con la clasificación de los invertebrados apoyada por Hyman.

Juan Pablo Bozzini, ex presidente del Centro de Estudiantes del Doctorado en Ciencias Naturales en el año 1956, contribuye con "Cien años de teoría de la selección natural", una reseña histórica del tema. Por su parte Richard Goldschmidt, de la Universidad de California, en Estados Unidos, escribe sobre "La evolución vista por un geneticista". Este artículo es la traducción de otro aparecido en el "American Scientist" y constituye un homenaje a su autor, recientemente fallecido en aquel entonces. 


\section{Número 15 (1959)}

En los número siguiente del tomo VI ya no aparecen avisos publicitarios si bien la Facultad de Ciencias Exactas y Naturales contribuye económicamente a su sustento.

Osvaldo Reig discute "Acerca de la ubicación de los estudios paleontológicos" para incluirlos sea en la Geología o en la Biología. Sus conclusiones sobre el tema señalan que la paleontología no es una rama de la geología, pues considera que los objetivos y métodos de trabajo son los mismos que los de la zoología y botánica y que entre éstas y la geología existe una disciplina de transición la bioestratigrafia.

Mario Bunge discurre acerca de "Qué es un problema científico" planteando en primer lugar ¿qué es un problema?, ¿en qué consiste el planteo científico de un problema? ¿hay problemas insolubles en ciencia? O ¿tiende disminuir el número de problemas?

Horacio Camacho se refiere a "un siglo de darwinismo" en una revisión de los cambios que ha sufrido el darwinismo desde la publicación del "Origin of species" aunque la selección natural permanece como el factor principal en la evolución.

Se incluye una conferencia de Richard F. Flint en el departamento de Ciencias Geológicas de la U.B.A., sobre "La glaciación pleistocena y las gravas tehuelches", a su juicio dos grandes e interesantes temas de la geología en la Argentina. Los sedimentos tehuelches por ser los de mayor difusión en el país y la glaciación por ser un tema de disputa entre varias corrientes de ideas. Finaliza este número con otro comentario al "Estudio sobre facies" de D. V. Nalivkin desarrollado por Sergio Archangelsky en que se resalta la importancia del estudios de las facies, para lo cual el especialista debe estar sólidamente preparado en geología, geomorfología y petrografía.

\section{Número 16 (1960)}

El número 16 del tomo VI sale a la luz en noviembre de 1960, un año después del anterior. Se inicia con un trabajo de Elías Balbinder sobre la "Estructura y acción de los genes" donde se refiere a los distintos elementos que contribuyen a la formulación de la teoría vigente entonces, con énfasis en los problemas de mutación en relación con la estructura del gen. Raúl Ringuelet traduce un trabajo de Claude Delamare Deboutteville sobre "Linajes marinos que han penetrado en las aguas subterránea continentales. Un problema de Biogeografía actual" Este estudioso francés en su recorrida por nuestro país detectó la presencia de Syncarida en aguas dulces de la Argentina.

Otro ex-presidente del Centro de Estudiantes, Guillermo Sarmiento, presenta un aporte al conocimiento de la "Estructura, organización y funcionamiento del meristema apical del vástago"; continua así una línea abierta en el número 14 con la difusión de los trabajos de nuevos egresados y sigue en el trabajo siguiente de Regina Levy sobre "Unidades estratigráficas" con un sumario de los conceptos modernos en estratigrafía y su uso en la Argentina.

El trabajo de H. Gaussen sobre "Juventud y evolución" discurre sobre el crecimiento en tamaño de las especies, de la ortogénesis, la evolución seudocíclica, el policiclismo y otros temas.

Este número incluye el "Manifiesto de Curitiba" referido a los efectos biológicos de las radiaciones y los graves riesgos que representan tanto para la actual como para las generaciones venideras, firmado por científicos, brasileños, uruguayos y argentinos en ocasión de realizarse la Primera reunión brasileña de Genética Humana y Cuarta semana de Genética que tuvo lugar en Curitiba, Brasil, entre el 10 y el 15 de noviembre de 1958. 


\section{Número 17 (1961)}

Un año más tarde en noviembre de 1961 aparece el último número de Holmbergia, el 17. Comienza con un artículo de Raúl A. Ringuelet sobre Florentino Ameghino al cumplirse el cincuentenario de su muerte. Sigue con otro sobre "Los efectos genéticos producidos por las explosiones atómicas", escrito por Newton Freire-Maia, donde el autor analiza los efectos inmediatos y de larga duración producidos por el incremento de las radiaciones debidas a las bombas atómicas detonadas al fin de la segunda guerra mundial y a experimentos nucleares posteriores a las que atribuye las mutaciones genéticas encontradas, por ejemplo, en los descendientes y los sobrevivientes de Hiroshima y Nagasaki. Alerta sobre el peligro que acecha a las futuras generaciones si continúan incrementándose las emanaciones radiactivas.

José Babini, quien fuera decano interventor de la Facultad entre 1955 y 1957, explica cómo eran "Las ciencias naturales en la época renacentista" y relata como Álvaro Alonso Barba, en 1590, describe por primera vez las reglas del beneficio de las menas de oro y plata por amalgamación.

Osvaldo A. Reig concluye este volumen de Holmbergia con un ensayo sobre "La paleontología de vertebrados en la Argentina. Retrospectiva y prospectiva", donde describe el desarrollo de los estudios de vertebrados fósiles desde el período pre-ameghiniano al post-ameghiniano, hasta 1961 y presenta sus ideas acerca de la organización del trabajo paleontológico.

\section{Conclusiones}

Con el número 17 de 1961 finaliza el ciclo de "Holmbergia". Cabe señalar que los años en que la revista no aparece coinciden con los de una universidad que, como es sabido, se vio marcada por una notable disminución de la calidad de la enseñanza además de una intolerancia manifiesta hacia opiniones democráticas, lo que llevó a muchos docentes a renunciar a sus actividades académicas como es el caso, emblemático, del Dr. Bernardo Houssay.

En el último número de la Revista se anunciaba la aparición de un número extraordinario de "Holmbergia" dedicado a "los problemas de Ecología regional, que Latinoamérica -arrasada por la explotación de rapiña- necesitará conocer a fondo para devolver a su pueblo subalimentado la productividad de sus ambientes naturales", un anticipo de nuestra actualidad. Lamentablemente nunca llegó a publicarse.

Una mirada al tipo de artículos registrados en la primera década de "Holmbergia" está revelando que los mismos son especialmente descriptivos y en parte reflejan el aislamiento científico de la Argentina durante la segunda guerra mundial. Los aires de renovación que se abren en las universidades argentinas a partir del golpe de estado de 1955 que derroca al general Perón, y en la Universidad de Buenos Aires, especialmente en la Facultad de Ciencias Exactas y Naturales a partir del decanato de José Babini y posteriormente el de Rolando García, también se ven reflejados en el enfoque y contenido de la Revista del Centro de Estudiantes de Ciencias Naturales, con mayor incidencia en enfoques novedosos y actualizados además de vinculados a los problemas nacionales del ambiente y la sociedad. 